\title{
Ramsay, Thomson, Burns et les peintres écossais
}

Ramsay, Thomson, Burns, and Scottish Painters

\section{Marion Amblard}

\section{(e) OpenEdition}

\section{Journals}

\section{Édition électronique}

URL : http://journals.openedition.org/etudesecossaises/946

DOI : 10.4000/etudesecossaises.946

ISSN : 1969-6337

\section{Éditeur}

UGA Éditions/Université Grenoble Alpes

\section{Édition imprimée}

Date de publication : 25 avril 2015

Pagination : 47-66

ISBN : 978-2-84310-296-7

ISSN : $1240-1439$

Référence électronique

Marion Amblard, «Ramsay, Thomson, Burns et les peintres écossais », Études écossaises [En ligne], 17 | 2015, mis en ligne le 25 avril 2016, consulté le 16 mars 2021. URL : http://

journals.openedition.org/etudesecossaises/946; DOI : https://doi.org/10.4000/etudesecossaises.946

(c) Études écossaises 


\section{Ramsay, Thomson, Burns et les peintres écossais}

Il a fallu attendre le XVIII ${ }^{\mathrm{e}}$ siècle pour que les liens entre littérature et peinture écossaises se développent ${ }^{1}$. La majeure partie des œuvres d'art pictural ayant été exécutées en Écosse avant la Réforme a été détruite lors des campagnes iconoclastes de la seconde moitié du XVI siècle. En effet, de cette période, il ne reste plus que quelques portraits de souverains écossais et un retable exécuté par le peintre hollandais Hugo van der Goes (v. 1420-1482) ainsi que plusieurs plafonds peints dans des demeures seigneuriales ${ }^{2}$. Seuls les livres liturgiques enluminés datant de l'époque médiévale, qui ont échappé aux iconoclastes, permettent d'établir un lien entre littérature et art pictural en Écosse. Si cette dernière ne fut pas dépourvue de peintres au XVII ${ }^{\mathrm{e}}$ siècle, ce ne fut qu'à partir du début du XVIII ${ }^{\mathrm{e}}$ siècle que peintres et hommes de lettres commencèrent à s'influencer mutuellement. Le poète Allan Ramsay (1684-1743) comptait parmi ses amis les plus proches les portraitistes William Aikman (16821731) et John Smibert (1688-1751) auxquels il dédia respectivement ses poèmes «Betty and Kate a Pastoral Farewell to Mr Aikman when He Went to London», «An Elegy in Memory of William Aikman» et «An Epistle to a Friend at Florence, in His Way to Rome » ${ }^{3}$. Ainsi que l'a rappelé son fils dans la biographie qu'il lui a consacré, Ramsay avait été attiré par la peinture et le dessin dès son plus jeune âge mais avait

1. Ce fut également à partir de cette période que se sont développés les liens entre les peintres et les penseurs écossais. Ces derniers ont fait appel aux talents des plus éminents portraitistes du XVIII ${ }^{\mathrm{e}}$ siècle : entre autres, Francis Hutcheson (1694-1747) et David Hume (1711-1776) ont posé pour Allan Ramsay; Henry Raeburn a peint le portrait de Hugh Blair (1718-1800) et de Thomas Reid (17101796). Les peintres ont été influencés par les écrits des penseurs contemporains; les théories esthétiques de Ramsay et ses portraits dénotent l'influence de Hutcheson et de Hume, tandis que les toiles de Raeburn évoquent les écrits de Reid. Sur les liens entre peinture et pensée esthétique au XVIII ${ }^{\mathrm{e}}$ siècle, voir D. Macmillan, Painting in Scotland. The Golden Age, Oxford, Phaidon Press, 1986, p. 18-39 et p. 74-136.

2. Sur les plafonds peints en Écosse, voir l'ouvrage de M. R. Apted, The Painted Ceilings of Scotland, 1550-1650, Édimbourg, Her Majesty's Stationery Office, 1966.

3. Smibert et Aikman ont tous deux peint un portrait d'Allan Ramsay (William Aikman, Allan Ramsay, 1722, National Gallery of Scotland, Édimbourg; John Smibert, Allan Ramsay, v. 1717, collection particulière). 
été contraint à renoncer à ses aspirations artistiques pour travailler tout d'abord comme perruquier ${ }^{4}$. Cet intérêt et son contact avec des peintres l'ont certainement incité à s'impliquer dans la création de l'Academy of Saint Luke, premier centre de formation artistique écossais qui fut fondé à Édimbourg en $1729^{5}$. Il a également encouragé la vocation de peintre de son fils, lui aussi nommé Allan Ramsay (1713-1784), et lui a offert son soutien lorsqu'il décida de partir étudier à Londres puis en Italie ${ }^{6}$. Ramsay le portraitiste compta parmi les plus éminents peintres britanniques du XVIII ${ }^{\mathrm{e}}$ siècle et fut le premier artiste écossais à être nommé au poste de Painter in Ordinary. Cependant, il aspira toute sa vie à être également reconnu en tant que poète et homme de lettres. Il publia douze essais abordant des sujets des plus divers tels que des théories esthétiques ou des questions politiques et il publia également un recueil de poésie. Après un accident en 1773, Ramsay ne peignit presque plus et, à sa mort en 1784, les articles parus dans les rubriques nécrologiques célébrèrent ses ouvrages littéraires plutôt que ses portraits ${ }^{7}$.

L'intérêt des peintres pour la littérature et l'attrait de la peinture pour les hommes de lettres écossais n'a pas cessé après le décès d'Allan Ramsay. Ainsi que le rappelle Sir William Allan (1782-1850) avec son tableau The Celebration of the Birthday of James Hogg représentant l'artiste attablé en compagnie de Walter Scott (1771-1832), Christopher North (1785-1854) et James Hogg (1770-1835), peintres et écrivains ont continué à se côtoyer. L'influence des romanciers et des poètes sur les peintres est devenue encore plus manifeste dès le début du XIX ${ }^{\mathrm{e}}$ siècle. Sir Walter Scott a notamment beaucoup contribué à l'épanouissement de l'école écossaise de peinture. Il a plus particulièrement aidé au développement de la peinture d'histoire grâce à ses romans fournissant un grand nombre de sujets aux peintres et en offrant son soutien et ses encouragements à son ami William Allan. Alors qu'Allan ne parvenait pas à trouver des acheteurs pour ses tableaux

4. A. Ramsay, «Life of A. R. by His Son Allan Ramsay the Painter », Edinburgh University Library, La. II. 212/42.

5. Allan Ramsay a fait partie des membres fondateurs de cette académie dont il signa la charte en compagnie de son ami James Norie (1684-1757), artiste à la tête d'une entreprise familiale de peinture décorative.

6. Sur la relation entre le poète Allan Ramsay et son fils portraitiste, voir I. G. Brown, Poet and Painter. Allan Ramsay Father and Son, 1684-1784, Édimbourg, National Library of Scotland, 1984.

7. Ainsi peut-on lire dans la rubrique nécrologique du Gentleman's Magazine du 10 août 1784 : «Died at Dover on his return from the continent in his 71 st year, Allan Ramsay Esq., Principal Portrait Painter to their Majesties. By his death, the polite and the literary world have sustained an irreparable loss, as few men have excelled him in correctness of taste, brilliancy of wit, or soundness of understanding. His publications are numerous and various; several of them are on political subjects in which he displayed much useful knowledge of the constitution of his country, for which he was a strenuous and disinterested advocate.» (Gentleman's Magazine and His Historical Chronicle, vol. 54, 10 août 1784 , p. 638 .) 
et songeait à quitter définitivement l'Écosse pour aller s'établir en Russie où il avait vécu pendant près de dix ans, Scott l'aida à surmonter ses problèmes financiers en le recommandant à son éditeur Archibald Constable qui l'employa dès 1818 pour réaliser treize illustrations des romans de Waverley $^{8}$. Ce fut également Scott qui suggéra à Allan de délaisser la peinture de genre représentant des scènes inspirées de son périple en Russie et de se spécialiser dans les sujets tirés de l'histoire écossaise. Ses toiles intitulées Heroism and Humanity: An Incident in the Life of Robert the Bruce et The Murder of Archbishop Sharp on Magus Moor, 1679 montrent qu'il suivit les conseils de l'écrivain et qu'il s'inspira des romans de Scott pour peindre des tableaux sur l'histoire de l'Écosse ${ }^{9}$. Allan ne fut pas le seul peintre à être marqué par les écrits de Scott puisque Catherine Gordon a estimé qu'entre 1805 et 1870 plus de trois cents peintres ayant exposé à la Royal Scottish Academy et à la Royal Academy se sont inspirés de Scott et ont exposé au total plus de mille tableaux ayant un thème tiré de ses ouvrages littéraires ${ }^{10}$. De même l'influence de Scott sur les peintres ne se limita pas à la Grande-Bretagne. En France, notamment, Eugène Delacroix (17981863) fut particulièrement sensible à son œuvre. Scott ne fut pas le seul homme de lettres écossais à avoir inspiré les peintres exerçant en Écosse puisque les poèmes d'Allan Ramsay et de Robert Burns (1759-1796) ont été le sujet de nombreux tableaux exécutés entre la fin du XVIII ${ }^{\mathrm{e}}$ siècle et le XIX ${ }^{\mathrm{e}}$ siècle.

Dans cet article, nous nous intéresserons plus précisément aux toiles des peintres de genre qui, pour la plupart, ont réalisé des œuvres illustrant des scènes tirées de The Gentle Shepherd de Ramsay ou des poèmes de Burns. Une étude de quelques tableaux nous permettra de montrer que l'on retrouve en peinture la dualité qui a caractérisé la littérature écossaise tout au long du XVIII ${ }^{\mathrm{e}}$ siècle : comme les poètes, les peintres ont insisté d'une part sur la spécificité de la culture écossaise et, d'autre part, ont célébré l'union avec l'Angleterre. Ces œuvres nous permettront également de constater que les peintres avaient, en commun avec Ramsay

8. Sur les illustrations des ouvrages de Scott par William Allan, voir R. J. Hill, Picturing Scotland through the Waverley Novels. Walter Scott and the Origins of the Victorian Illustrated Novel, Ashgate, Farnham and Burlington, 2010, p. 99-132.

9. Ainsi qu'Allan l'a rappelé dans le catalogue de l'exposition annuelle de la Royal Scottish Academy de 1840, il s'était inspiré d'une anecdote de Tales of a Grandfather (1828-1830) pour peindre cette toile (The Exhibition of the Royal Scottish Academy of Painting, Sculpture, and Architecture, 1840, the Fourteenth, Édimbourg, H. and J. Pillans, 1840, p. 13). Selon Hill, Allan se serait inspiré du roman Old Mortality (1816) lorsqu'il a peint la scène du meurtre de l'archevêque Sharp : «the major influence for this painting was Scott's by now famous Old Mortality (1816)» (Picturing Scotland through the Waverley Novels, ouvr. cité, p. 101).

10. G. Gordon, The Lamp of Memory - Scott and the Artist, Derby, Derbyshire Museum Service, 1979, p. II. 
et Burns, un intérêt pour les traditions écossaises et la vie quotidienne de leurs compatriotes. Pour finir nous rappellerons qu'à l'instar des poèmes de Burns les œuvres de David Wilkie (1785-1841), qui a abordé des thèmes propres à la culture écossaise, ont su franchir les frontières de l'Écosse pour susciter l'admiration et inspirer quelques-uns des plus éminents peintres de l'Europe continentale.

La signature du traité d'Union des Parlements anglais et écossais en 1707 fut un événement marquant dans l'histoire de l'Écosse puisque, après avoir lutté durant des siècles pour rester indépendante, elle renonçait à son autonomie. La fusion parlementaire ne s'y accomplit pas sans heurt et durant la première moitié du siècle il y eut plusieurs révoltes armées. Une partie des Écossais s'était opposée à une telle union et, jusqu'en 1746, date de la dernière rébellion jacobite, ils espérèrent pouvoir retrouver leur indépendance. La sévère défaite infligée aux troupes de Charles-Édouard Stuart mit fin à tout espoir et les Écossais durent se résoudre à accepter cette union ou, du moins, à s'en accommoder avant d'en devenir de fervents partisans à l'issue des guerres napoléoniennes. Le traité de 1707 mettait en place une union politique et économique entre l'Angleterre et l'Écosse, mais garantissait à cette dernière le maintien de l'Église presbytérienne ainsi que des systèmes judiciaire et éducatif qui lui étaient propres. Même lorsqu'ils se sont réconciliés avec l'idée d'être unis avec les Anglais au sein de la Grande-Bretagne, les Écossais ont su préserver leur propre identité culturelle. L'Union les a incités à se démarquer par crainte d'une assimilation intégrale dans une Grande-Bretagne dominée politiquement et économiquement par l'Angleterre. Cette volonté de se distinguer a été en partie à l'origine de l'effervescence culturelle qui a animé l'Écosse à partir de la seconde moitié du XVIII siècle. Si certains intellectuels écossais, tel David Hume (17111776), étaient gênés par leur accent au point de fonder en 1760 The Society for Promoting the Reading and Speaking of the English Language afin de prendre des cours de phonétique et de langue anglaise pour amoindrir leur accent et ne plus employer de scotticismes, d'autres ont au contraire revendiqué la spécificité linguistique et culturelle de l'Écosse en composant des poèmes en scots plutôt qu'en anglais. Alors qu'au XVII siècle la plupart des poètes écossais avait écrit en anglais, Allan Ramsay fut à l'origine d'un renouveau d'intérêt pour la poésie en langue vernaculaire. Il composa et rassembla de nombreux poèmes et chants traditionnels dans deux recueils, Tea-Table Miscellany et Ever Green, parus en 1721 et en 1724, et écrivit en 1725 une comédie pastorale, The Gentle Shepherd, qui lui valut un immense succès en Écosse et en Angleterre : Alexander Pope (16881744) et John Gay (1685-1732) comptaient notamment parmi ses plus fervents admirateurs. Durant la seconde moitié du XVIII ${ }^{\mathrm{e}}$ siècle la poésie 
en scots continua à être des plus populaires et Robert Fergusson (17501774) ainsi que Robert Burns en furent les deux principaux représentants. En plus d'affirmer l'originalité linguistique et culturelle de l'Écosse, les poèmes en scots peuvent être associés à un sentiment nationaliste et antiunioniste qui reflétait l'attitude d'une partie de la population écossaise vis-à-vis de l'Union des Parlements au XVIII ${ }^{\mathrm{e}}$ siècle. Ainsi dans ses poèmes sur les jacobites tels «O'er the Water to Charlie» et «Charlie he's My Darling», et dans «Such a Parcel of Rogues in a Nation» avec sa célèbre strophe :

O would, or I had seen the day

That Treason thus could sell us,

My auld grey head had lien in clay,

Wi' Bruce and loyal Wallace!

But pith and power, till my last hour,

I'll mak this declaration:-

'We're bought and sold for English gold'-

Such a parcel of rogues in a nation! (v. 17-24)

Burns se lamente de la perte de l'indépendance de l'Écosse et condamne les membres du Parlement écossais qui ont accepté de signer le traité d'union. Si dès le début du XVIII ${ }^{\mathrm{e}}$ siècle les poètes n'ont pas hésité à s'exprimer sur l'Union de 1707 et n'ont pas caché leur sentiment nationaliste, ce ne fut que durant la seconde moitié du XVIII ${ }^{\mathrm{e}}$ siècle que les peintres ont réellement pu commencer à en faire de même. Thomas Warrender (1662- v. 1715), qui a peint vers 1708 un trompe l'œil représentant un porte-lettres dans lequel il évoque son soutien pour l'Union, est une exception ${ }^{11}$. Avant la seconde moitié du XVIII ${ }^{\mathrm{e}}$ siècle les peintres n'avaient en effet guère la possibilité de faire part de leurs opinions : jusque-là le marché de l'art écossais était relativement limité et, tant que les jacobites représentaient une menace pour la stabilité de la GrandeBretagne, les peintres pouvaient difficilement afficher leurs opinions politiques sans mettre en jeu leur carrière. Certains artistes tels John Alexander (1686-1766) et son fils Cosmo (1724-1772) ont été contraints à l'exil pendant plusieurs années en raison de leur soutien aux Stuart. Dès lors que les rebellions jacobites ont été définitivement maîtrisées, que les commandes de tableaux ont commencé à augmenter et que des genres picturaux autres que le portrait se sont développés, les peintres ont pu plus librement faire part de leurs sentiments concernant l'union anglo-

11. Selon Murdo Macdonald, dans ce tableau, le peintre «seems to be both invoking the Union as a defence of Presbyterianism and reminding those who held power in the Union to honour the letter of the Union and defend Presbyterianism» (M. Macdonald, Scottish Art, Londres, Thames and Hudson, 2000, p. 51). 
écossaise. Une étude des productions picturales datant des XVIII $^{\mathrm{e}}$ et XIX ${ }^{\mathrm{e}}$ siècles montre que peintres et poètes partageaient les mêmes points de vue et que leurs opinions ont évolué de façon comparable. À partir de la seconde moitié du XVIII ${ }^{\mathrm{e}}$ siècle, on peut distinguer deux types de sentiments à travers les tableaux des peintres écossais avec d'une part des toiles peintes dans un style classique alors en vogue en Angleterre et sur le continent européen célébrant l'union anglo-écossaise et, d'autre part, des tableaux exécutés dans un style sobre et réaliste qui soulignent l'originalité culturelle de l'Écosse. Toutefois, à la différence des poètes, les peintres n'ont pas exprimé un sentiment anti-unioniste, ceci s'explique peut-être par le fait que lorsqu'ils réalisèrent leurs œuvres la population écossaise avait accepté l'idée d'être unie à l'Angleterre. De plus, jusqu'à la première moitié du XIX siècle ils allaient travailler à Londres, principal centre artistique de Grande-Bretagne, et ne pouvaient pas proposer à leurs mécènes anglais des tableaux critiquant l'union des Parlements. En revanche, comme les poèmes en scots, les toiles révèlent une nostalgie et un attachement profond à la culture et aux traditions écossaises.

Cette dualité unionisme/nationalisme qui caractérisa la poésie et la peinture écossaises est particulièrement apparente lorsque l'on compare les scènes de genre aux toiles des peintres de paysages exécutées avant 1830. Par la suite, cette opposition a disparu et a été remplacée par un sentiment alliant pro-unionisme et nationalisme qui devint manifeste dans les œuvres littéraires et picturales de cette période. La série de tableaux d'Alexander Nasmyth (1758-1840) peinte au début du XIX siècle et représentant des vues de la ville d'Édimbourg est un véritable hymne en l'honneur de la Grande-Bretagne, à l'instar des poèmes de James Thomson (1700-1748) ${ }^{12}$. Dans Princes Street with the Commencement of the Building of the Royal Institution le peintre montre la prospérité de la capitale écossaise qui est indissociable de son alliance avec l'Angleterre. Dans cette toile, l'artiste se place à l'ouest d'Édimbourg pour offrir une vue du centre-ville. Même si l'architecture et les bâtiments élevés de la ville médiévale contrastent avec le style néoclassique de la nouvelle ville, tout dans la composition du tableau donne une impression d'harmonie et de complémentarité. En effet, la composition est symétrique : la ligne d'horizon est au centre du tableau et le plan se situant en dessous de cette ligne se compose de deux parties avec la nouvelle ville se trouvant sur la gauche de la toile et la vieille ville sur la droite. Avec les nombreux personnages se trouvant

12. Sur les vues d'Édimbourg par Nasmyth, voir M. Amblard, «Édimbourg vue par Alexander Nasmyth : une représentation de l'identité écossaise au début du dix-neuvième siècle», dans T. Tran, R. Findlay et W. Findlay (éds), Vivre la ville en Écosse, GRAAT, n 32, Tours, Presses universitaires François Rabelais, 2005, p. 89-108. 
dans Princes street et les ouvriers occupés à construire la Royal Institution, le peintre suggère la vitalité de la capitale écossaise et rappelle que, depuis 1707, elle s'est rapidement développée. La vieille ville est dominée par la cathédrale Saint Giles tandis que la nouvelle ville est surplombée par Calton hill; le regard de l'observateur est attiré sur le Nelson Monument qui constitue le point de fuite de deux lignes diagonales ascendantes. Cette tour et l'ensemble des monuments de Calton hill ont été construits en mémoire des soldats qui se sont battus contre les troupes de Napoléon; les rues de la nouvelle ville ont été baptisées en l'honneur des monarques britanniques et de la dynastie des Hanovre. Le peintre célèbre ainsi la Grande-Bretagne qui permet à l'Écosse de prospérer mais rappelle aussi que cette dernière contribue à l'hégémonie britannique. Cette toile de Nasmyth dégage un souffle patriotique comparable à celui qui émane des poèmes de James Thomson; le peintre s'accorde avec le poète pour louer les bienfaits de l'union avec l'Angleterre et la contribution de l'Écosse à ce partenariat :

Oh! Is there not some patriot, in whose power

That best, that godlike Luxury is plac'd,

Of blessing thousands, thousands yet unborn,

Thro' late prosperity? some, large of soul,

Tho chear dejected industry? to give

A double harvest to the pining swain,

And teach the lab'ring hand the sweets of toil?

How by the finest art the native robe

To weave; how, white as hyperborean snow,

To form the lucid lawn; with venturous oar

How to dash wide the billow: nor look on,

Shamefully passive, while Batavian fleets

Defraud us of the glittering finny swarms,

That heave our friths, and crowd upon our shores;

How all-enlivening trade to rouse, and wing

The prosperous sail, from every growing port,

Uninjur'd, round the sea-encircled globe;

And thus, in soul united as in name,

Bid Britain reign the mistress of the deep? ${ }^{13}$ (v. 910-928)

13. Le tableau de Nasmyth évoque aussi «Rule Britannia» de Thomson dans lequel le poète explique que l'alliance de l'Écosse et de l'Angleterre permet d'affirmer la grandeur de la GrandeBretagne face à ses rivaux et contribue à sa richesse : «The nations not so blest as thee, / Must in their turns to tyrants fall; / While thou shalt flourish great and free, / The dread and envy of them all. / Rule, Britannia, rule the waves; / Britons never will be slaves. » (v. 7-12) 
Nasmyth ne fut pas le seul peintre écossais dont les tableaux évoquent les poèmes de Thomson. Auparavant, Jacob More (1740-1796) avait peint plusieurs toiles représentant les chutes de la Clyde qui rappellent une description de cascades dans «Summer» de Thomson (v. 592-606), mais aucune de ses œuvres ne dénotent un sentiment patriotique. Comme Thomson l'avait fait avant lui, More décida de quitter son pays natal pour sa carrière et peignit des paysages italiens ou imaginaires plutôt que des vues d'Écosse; si le poète a choisi de composer en anglais et non en scots, More - et il en fut de même pour Nasmyth à la fin du XVIII ${ }^{\mathrm{e}}$ siècle - a opté pour un style classique évoquant celui de Claude Lorrain (1600-1682) plutôt que d'essayer de s'affranchir des influences étrangères pour développer sa propre manière. Au contraire, à l'instar des poètes en scots, les peintres de genre ont préféré des sujets spécifiques à l'Écosse et ont abordé ces thèmes dans des toiles exécutées dans un style qui devint caractéristique de l'école écossaise comme c'est le cas, par exemple, de John Phillip (1817-1867) avec son tableau Baptism in Scotland qui représente une famille de paysans rassemblée autour d'un pasteur de l'église presbytérienne alors qu'il baptise un enfant. Dans cette scène d'intérieur la lumière, qui provient de la fenêtre à l'arrière-plan attire l'attention de l'observateur sur le pasteur, l'enfant qui est baptisé et ses parents qui se trouvent au centre du tableau. Ainsi que le rappelle Brown, le baptême est un des deux sacrements de l'Église d'Écosse ${ }^{14}$ et dès le début du XIX ${ }^{\mathrm{c}}$ siècle cette cérémonie n'avait plus lieu à l'église, elle était devenue un événement privé célébré par le pasteur dans la demeure des paroissiens. Le style mimétique et le coloris du tableau dominé par les teintes brunes évoquent les toiles de Wilkie, qui aimait particulièrement peindre des scènes d'intérieur, et s'inscrit dans la lignée des œuvres picturales écossaises rendant hommage à l'Église presbytérienne et rappelant son importance dans la société écossaise. Avant Phillip plusieurs peintres avaient exécuté des toiles ayant pour sujet l'Église presbytérienne : George Harvey (1806-1876) avait notamment peint Catechising in a Scottish School et The Christening ${ }^{15}$, tandis qu'Alexander Carse (v. 1770-1843) avait imaginé une scène du XVII ${ }^{\mathrm{e}}$ siècle rassemblant des Covenanters autour d'un pasteur dans Covenanters in a Glen. Les peintres ont également célébré l'originalité et la richesse de la culture écossaise en peignant des scènes inspirées de poèmes en scots.

14. Ainsi que le précise Brown : «apart from the Lord's supper, the only sacrament recognised in Scottish Presbyterianism was baptism» (S. J. Brown, «Beliefs and Religions», dans T. Griffiths et G. Morton (éds), A History of Everyday Life in Scotland, 1800 to 1900, Édimbourg, Edinburgh University Press, 2010, p. 126).

15. George Harvey a également réalisé une série de scènes de genre historique montrant des Covenanters. 
Plusieurs spécialistes ont comparé les tableaux des peintres de genre aux poèmes en langue vernaculaire composés par Ramsay et Burns. Entre autres, les toiles de Thomas Faed (1826-1900) ont été associées aux œuvres de Burns et David Allan a été comparé à Ramsay et à Burns dont il illustra les poèmes ${ }^{16}$. Allan fut le premier artiste écossais à s'inspirer des poèmes en scots et réalisa plusieurs illustrations pour des poèmes de Burns ainsi que douze gravures pour accompagner une édition de The Gentle Shepherd datant de 1788. Les gravures produites pour l'ouvrage de Ramsay lui ont assuré une grande renommée en Écosse et suscitèrent l'admiration de Burns qui écrivit dans une de ses lettres:

Do you know Allan? He must be a man of very great genius. - Why is he not more known? Has he no Patrons, do Poverty's cold wind \& crushing rain bat on him? I once and but once got a glance of that noble edition of the noblest Pastoral in the world, \& dear as it was; I mean dear as to my pocket, I would have bought it; but I was told that it was printed and engraved for Subscribers only. He is the only artist who has hit genuine Pastoral costume ${ }^{17}$.

The Gentle Shepherd de Ramsay et les poèmes de Burns ont en partie contribué au développement et à l'originalité de la peinture de genre en fournissant de nombreux sujets aux peintres. Ainsi que le précise Allan Cunningham dans sa biographie du peintre, Sir David Wilkie vouait une grande admiration à Allan Ramsay et exécuta à l'âge de dix-sept ans une première toile, Patie Disbelieves Sir William's Prophecy from 'The Gentle Shepherd', montrant Sir William prédisant l'avenir à son fils alors que celui-ci ignore encore les liens de parenté qui les unissent ${ }^{18}$. Par la suite, il continua à s'inspirer de cette comédie pastorale et peignit notamment deux autres tableaux, Roger Piping to Jenny, from 'The Gentle Shepherd' et The Cottage Toilet' ${ }^{19}$. The Cottar's Saturday Night et Duncan Gray de Wilkie sont quant à eux tirés

16. Un article de l'Encyclopedia Americana de 1886 affirme : «It has been truly said that Thomas Faed has done for Scottish art what Robert Burns did for Scottish song. He has made it attract universal interest and command universal respect. " (Cité dans M. McKerrow, The Faeds, a Biography, Édimbourg, Canongate, 1982, p. 108.) Cursiter, les Irwin et, plus récemment, Duncan Macmillan ont tous associé les œuvres d'Allan aux poèmes de Ramsay et de Burns. S. Cursiter, Scottish Art to the Close of the Nineteenth Century, Londres, George G. Harrap and Co., 1949, p. 50. D. et F. Irwin, Scottish Painters at Home and Abroad 1700-1900, Londres, Faber and Faber, p. 118-121. D. Macmillan, Scottish Art 1460-2000 [1990], Édimbourg et Londres, Mainstream Publishing, 2000, p. 130-132.

17. R. Burns, The Letters of Robert Burns, J. De Lancey Ferguson (éd.), vol. 2, Oxford, Clarendon Press, 1931, p. 237-238.

18. A. Cunningham, The Life of Sir David Wilkie: With His Journals, Tours, and Critical Remarks; and a Selection of His Correspondence, vol. 1, Londres, John Murray, 1843, p. 50.

19. Peu avant son décès, Wilkie prévoyait de réaliser des œuvres pour illustrer une nouvelle édition de la comédie pastorale ainsi que le précise son biographe : «Wilkie himself, towards the close of a short and brilliant career, informed me it was his purpose to embellish [The Gentle Shepherd].» (The Life of Sir David Wilkie, vol. 1, ouvr. cité, p. 55.) 
des poèmes éponymes de Burns. Certaines œuvres de Burns ont plus particulièrement inspiré les peintres, c'est le cas notamment de «Tam o'Shanter» qui raconte l'histoire de Tam qui a passé la soirée à boire dans un pub d'Ayr et a des hallucinations alors qu'il rentre chez lui. Entre autres, Walter Geikie (1795-1837), John Drummond (1802-1889) et John Faed (1819-1902), qui a exécuté une série d'illustrations pour ce poème parue dans une édition de $1855^{20}$, ont choisi de représenter le même passage, lorsque la sorcière Nannie est sur le point de rattraper Tam fuyant et chevauchant sa jument Maggie :

Now, do thy speedy utmost, Meg,

And win the key-stane of the brig;

There, at them thou thy tail may toss,

A running stream they dare na cross.

But ere the key-stane she could make,

The fient a tail she had to shake!

For Nannie, far before the rest,

Hard upon noble Maggie prest,

And flew at Tam wi' furious ettle;

But little wist she Maggie's mettle -

Ae spring brought off her master hale,

But left behind her ain grey tail;

The carlin claught her by the rump,

And left poor Maggie scarce a stump. (v. 209-222)

Toutefois, si les peintres ont été comparés aux poètes, ce n'est pas seulement parce qu'ils ont exécuté des tableaux et des gravures illustrant des scènes tirées des poèmes de Ramsay et de Burns. En effet, leurs œuvres présentent des affinités stylistiques et thématiques. Ainsi que l'a souligné l'historien de l'art James Caw, Allan a traité de thèmes comparables à ceux qui avaient inspiré les poètes en langue vernaculaire du XVIII ${ }^{\mathrm{e}}$ siècle :

Ramsay had reawakened the nation's interest in its own poetry, Fergusson had given vivid expression to some phases of contemporary life, Burns had just added the crowning glory of his song, and David Allan [...] led the way towards similar themes in pictorial art. (Caw, 1975, p. 51)

En Écosse, Allan fut le premier à se spécialiser dans la scène de genre et ses tableaux, aquarelles et gravures ont inspiré plusieurs générations d'artistes. Outre les poèmes en scots, Allan et les peintres du XIX ${ }^{\mathrm{c}}$ siècle

20. Faed a également réalisé des œuvres pour illustrer une édition de The Cottar's Saturday Night en 1853. Ce passage du poème de Burns a également été illustré par Eugène Delacroix en 1825 (Tam o' Shanter, Nottingham City Museums and Galleries, Nottingham). 
comptaient parmi leurs sujets de prédilection les traditions écossaises et la vie quotidienne de leurs compatriotes qui figuraient également parmi les principales sources d'inspiration des poètes en langue vernaculaire. Allan a notamment été à l'origine de deux thèmes récurrents dans la peinture de genre écossaise au XIX ${ }^{\mathrm{e}}$ siècle : il fut le premier artiste écossais à représenter un mariage traditionnel appelé penny wedding ${ }^{21}$ et à donner une place centrale aux musiciens accompagnant les festivités. Dans son aquarelle représentant un penny wedding, la composition est dominée par les violonistes se trouvant sur la gauche de la composition. William Home Lizars (1788-1859), Alexander Carse et David Wilkie, qui ont tous exécuté une toile montrant un mariage traditionnel, ont eux aussi accordé une place importante aux musiciens et, à l'exception de Lizars qui les a représentés au premier plan à droite, les peintres ont suivi l'exemple d'Allan et les ont placés en position dominante sur la gauche du tableau. Dans l'aquarelle d'Allan le musicien représenté est le célèbre violoniste Niel Gow qu'il avait déjà peint en 1780 dans une scène montrant un mariage dans les Highlands ainsi que dans un portrait le représentant avec son frère Donald, lui aussi musicien. Niel Gow, qui a posé pour le portraitiste Sir Henry Raeburn (1756-1823) en 1792, figure également sur le Penny Wedding de Wilkie qui rend ainsi hommage au musicien et à Allan dont il s'inspira pour ce tableau. Le violoniste fut également célébré par Burns qui le surnommait le «king o'rant and reel» (v. 1) dans «To Mr Gow, Visiting Dumfries», poème qu'il composa quelques années après qu'Allan ait peint le Highland Wedding et le double portrait des frères Gow. Les œuvres d'Allan, de Wilkie et de Raeburn évoquent les liens entre peinture, poésie et musique en Écosse et rendent hommage à la culture populaire. Les traits rugueux de l'aquarelle d'Allan, les tons bruns de la toile de Wilkie et l'arrière-plan dépouillé du portrait de Raeburn évoquent la simplicité et l'attachement aux traditions et font écho à la langue vernaculaire utilisée par Burns et à la musique de Gow, qui composa des airs célèbres du répertoire de la musique écossaise traditionnelle. Si les poètes en scots dédièrent plusieurs poèmes à la musique, tels «Bagpipes no Music» de Ramsay et «A Fiddler in the North» de Burns, ce fut aussi un thème récurrent dans les scènes de genre du XIX ${ }^{\mathrm{e}}$ siècle : entre autres, parmi les œuvres de Wilkie The Blind Fiddler, Roger Piping to Jenny, from 'The Gentle Shepherd' et The Bag-Piper ont pour personnages principaux

21. Grey Graham a expliqué à propos de ce type de mariage : «It was the custom of the country for friends and neighbours to subscribe money - originally a penny each - to provide food, drink, and fiddler.» (H. Grey Graham, The Social Life of Scotland in the Eighteenth Century, Londres, A. and C. Black, 1901, p. 327.) Le premier penny wedding fut peint par le Hollandais Jacob de Wet (1610v. 1671). 
des musiciens; durant la seconde moitié du XIX ${ }^{\mathrm{e}}$ siècle des musiciens sont également apparents dans The Music Room de John Phillip, A Musician's Reverie et $A$ Song without Words de John Pettie (1839-1893)22. Poètes et peintres de genre avaient aussi en commun un intérêt pour les fêtes de village qui ne manquaient pas d'animation ainsi que le suggère Wilkie dans sa toile Pitlessie Fair. Dans cette œuvre exécutée en 1804, le peintre a représenté une vue de la fête de son village natal dans le comté de Fife. Ce tableau comprend plus de cent quarante personnages et, comme l'a rappelé son biographe, la plupart était des paroissiens que Wilkie avait croqués à l'église pendant les sermons prêchés par son père, le révérend David Wilkie ${ }^{23}$. Certains personnages sont occupés à bavarder ou à négocier tandis que d'autres mangent et boivent; des jeunes hommes courtisent des jeunes filles; au premier plan, des enfants jouent ou se battent. Cette atmosphère de confusion qui émane du tableau évoque «The Holy Fair» poème de Burns où tous les villageois se réunissent pour participer aux festivités :

Then I gaed hame at crowdie-time,

An' soon I made me ready;

For roads were clad, frae side to side,

Wi' mony a weary body

In droves that day.

Here farmers gash, in ridin graith,

Gaed hoddin by their cotters;

There swankies young, in braw braid-claith,

Are springing owre the gutters.

The lasses, skelpin barefit, thrang,

In silks an' scarlets glitter;

Wi' sweet-milk cheese, in mony a whang,

An' farls, bak'd wi' butter,

Fu' crump that day. (v. 48-61)

Si les poètes et les peintres se sont inspirés de la culture écossaise ou du quotidien de leurs compatriotes, ceci ne limita pas leur succès à l'Écosse. Parmi les poètes, Burns et James Macpherson (1736-1796) avec ses poèmes d'Ossian, ont joui d'une renommée considérable en Angleterre et sur le

22. L'historien de l'art Duncan Macmillan s'est intéressé au thème de la musique dans la peinture écossaise des XVIII ${ }^{\mathrm{e}}$ et XIX ${ }^{\mathrm{e}}$ siècles dans un de ses articles. Voir D. Macmillan, «Old and Plain: Music and Song in Scottish Art», dans J. Cowan (éd.), The People's Past, Édimbourg, Polygon, 1980, p. 131144. Selon Macmillan ce thème a servi à démarquer l'école écossaise de celles du continent car «during this period of a century [from the mid-eighteenth century to 1849] when popular music was absent from the imagery of the rest of European art it was central to that of art in Scotland» (ibid., p. 134).

23. The Life of Sir David Wilkie, vol. 1, ouvr. cité, p. 60. 
continent européen, tandis que les scènes de genre écossaises de David Wilkie lui assurèrent une notoriété qui allait au-delà des frontières de son pays natal et inspirèrent quelques-uns de ses plus éminents contemporains $^{24}$.

En observant Roger Piping to Fenny, from 'The Gentle Shepherd' de Wilkie, Hogg ne put contenir son émotion et écrivit :

I got only one short look of it; but I saw nature so beautifully depicted, that in spite of all I could do the tears burst from my eyes, and the impressions made by it is as powerful at this moment as it was then. [...] There was never anything of the kind made such an impression on $\mathrm{me}^{25}$.

À l'instar de Hogg, le public et les connaisseurs anglais ont plus particulièrement apprécié les œuvres que Wilkie réalisa avant 1825 et représentant le plus souvent des scènes écossaises ${ }^{26}$. Pitlessie Fair, qu'il exposa peu après s'être installé à Londres en 1805, attira immédiatement l'attention des collectionneurs. Après avoir vu ce tableau, le comte de Mansfield lui commanda en 1805 Village Politicians, première œuvre qu'il exposa à la Royal Academy l'année suivante. Cette toile fut des plus remarquées et, grâce à celle-ci, Wilkie connut un succès immédiat. L'année suivante The Blind Fiddler, exposé à la Royal Academy, confirma son succès et, dès lors, il a joui d'une forte popularité auprès de quelques-uns des plus grands collectionneurs d'œuvres d'art en Angleterre qui appréciaient l'humour et le fini minutieux de ses tableaux leur rappelant les œuvres des peintres de genre hollandais et flamands du XVII ${ }^{\mathrm{e}}$ siècle. Wilkie bénéficia d'un si grand nombre de commandes qu'il put se consacrer uniquement à la peinture de genre dès 1810, alors qu'auparavant il avait dû peindre des portraits afin de subvenir à ses besoins. La demande pour ses tableaux fut si importante que même ses plus prestigieux clients durent parfois attendre

\footnotetext{
24. Alexander Runciman jouissait lui aussi d'une importante notoriété en Grande-Bretagne à la fin du XVIII ${ }^{e}$ siècle après avoir exécuté en 1772 vingt et une peintures murales sur le thème des poèmes de James Macpherson dans une pièce de la demeure de Sir James Clerk à Penicuik. Le peintre Henri Fuseli (1741-1825) comptait parmi ses plus fervents admirateurs. Dans une de ses lettres, Wilkie expliquait : «Mr Fuseli [...] questioned me about our artists in Edinburgh [...] He admired the works of the celebrated Runchiman and asked if I had ever seen his Ossians Hall at Pennycook.» («Wilkie», National Library of Scotland, MS. 9835)

25. The Art-Union, mai 1839, no 4, p. 74.

26. Le tableau intitulé Chelsea Pensioners Reading the Waterloo Despatch (1822, The Wellington Collection Apsley House, Londres) fut une exception. Cette œuvre représentant des vétérans de l'armée britannique apprenant la victoire de Wellington contre les troupes de Napoléon remporta un immense succès et des barrières furent même dressées devant le tableau afin de le protéger lorsqu'il fut exposé en 1822. 1825 fut une année charnière dans la carrière de Wilkie : alors qu'il souffrait de dépression il décida de quitter la Grande-Bretagne; il voyagea à travers l'Europe jusqu'en 1828 et, à son retour en Grande-Bretagne, il délaissa les scènes de genre écossaises pour peindre essentiellement des toiles inspirées de son périple sur le continent.
} 
plusieurs années avant d'obtenir une œuvre : ce fut notamment le cas du roi Georges IV qui reçut The Penny Wedding en 1819 alors que le tableau avait été commandé en 1813. Ceci n’empêcha pas le monarque de continuer à faire l'acquisition de ses toiles et en 1829 et en 1830 il ne lui acheta pas moins de quatre tableaux ${ }^{27}$. Outre Georges IV, Wilkie comptait parmi ses clients le duc de Wellington, Sir George Beaumont, le duc de Gloucester ainsi que le roi de Bavière qui lui commanda The Reading of the Will en 1820. Les membres de la Royal Academy ont eux aussi rapidement reconnu le talent de Wilkie et l'élurent Associé en novembre 1809, puis Académicien en février $1811^{28}$. Alors au sommet de sa carrière, Wilkie se vit décerner de prestigieuses distinctions par Georges IV qui le nomma au poste de King's Limner for Scotland après le décès de Sir Henry Raeburn en 1823 puis, lorsque Thomas Lawrence (1769-1830) décéda en 1830, il lui attribua le poste de Painter in Ordinary. À l'accession de Guillaume IV en 1830, Wilkie continua à bénéficier régulièrement du mécénat royal; son titre de Painter in Ordinary fut renouvelé et il fut même fait chevalier en 1836. En France, les talents de Wilkie furent appréciés par le roi Louis-Philippe ${ }^{\text {er }}$ qui le nomma chevalier de la légion d'honneur en avril $1841^{29}$ et par des peintres tels Géricault (1791-1824) et Delacroix. Tous deux ne manquèrent pas d'aller à son atelier lorsqu'ils séjournèrent à Londres : Géricault rendit visite à Wilkie en 1821 et fut admiratif de Chelsea Pensioners Reading the Waterloo Despatch qui était encore dans l'atelier du peintre ${ }^{30}$; pour sa part, Delacroix expliqua dans son journal que sa rencontre avec Wilkie en 1825 lui permit de découvrir le talent de ce dernier : "J'ai été chez M. Wilkie, et je ne l'apprécie que depuis ce moment. Ses tableaux achevés m'avaient déplu, et dans le fait, ses ébauches et ses esquisses sont au-dessus de tous les éloges ${ }^{31}$.» Quelques années plus tard, alors qu'il était de passage à Paris, ce fut Wilkie qui rendit visite à Delacroix et celui-ci fit à nouveau part de son admiration à la vue d'une étude préparatoire pour John Knox Preaching before the Lords of the Congrega-

27. Le monarque acheta The Defence of Saragossa (1828, collection de Sa Majesté la Reine Elisabeth II) The Spanish Posada (v. 1828, collection de Sa Majesté la Reine Elisabeth II), The Guerilla's Departure (v. 1828, collection de Sa Majesté la Reine Elisabeth II) et The Guerilla's Return (1830, collection de Sa Majesté la Reine Elisabeth II).

28. Toutefois, lorsqu'il commença à exposer à Londres, certains artistes n'approuvèrent pas la popularité des peintures de Wilkie. Comme Marks l'a precise : «[Village Politicians] was shown against the advice of several Academicians, notably John Flaxman "who as might naturally be supposed from the classical and antique refinement of his taste, had but little relish for rude nature, strong character, and rustic humour, held out small hopes of success from the experiment, advising the adoption of a totally different line of art for the neophytes' pursuit".» (A. S. Marks, «David Wilkie's 'Letter of Introduction'», The Burlington Magazine, n 110 , mars 1968, p. 126.)

29. Voir la lettre officielle conservée à la National Library of Scotland, Acc. 10063.

30. J. Sagne, Géricault, Paris, Fayard, 1991, p. 251.

31. Cité dans E. Chesneau, La Peinture anglaise, New York, Parkstone Press International, 2012, p. 59. 
tion, 10 June $1559^{32}$. Les œuvres de Wilkie ne semblent toutefois pas avoir influencé les peintres français; en revanche, elles marquèrent si profondément les artistes anglais que le graveur Abraham Raimbach n'hésita pas à affirmer que Wilkie était le chef de file de l'école anglaise de la peinture de genre au XIX ${ }^{\mathrm{e}}$ siècle $^{33}$. Dès lors qu'elles furent exposées en Angleterre, les scènes de genre écossaises de Wilkie eurent un impact immédiat et durable sur les peintres. Entre autres, Edward Bird (1772-1819) l'imita et fut son principal rival jusque vers 1810. Des artistes tels que l'Irlandais William Mulready (1786-1863) et William Collins (1788-1847), qui étaient des amis de Wilkie, se sont eux aussi inspirés du peintre écossais pour plusieurs œuvres ${ }^{34}$. Wilkie et Collins firent connaissance alors qu'ils étudiaient à la Royal Academy. Tout au long de leur vie, les deux hommes se sont régulièrement côtoyés et Collins avait tellement d'estime pour son ami qu'il baptisa son fils Wilkie. Dans la biographie qu'il consacra à son père, Wilkie Collins (1824-1889) rappela que Wilkie avait exercé une influence profonde sur son père ${ }^{35}$. Sir Edwin Landseer (1802-1873), qui était de son vivant un des peintres les plus prisés de Grande-Bretagne, a lui aussi peint une scène de genre intitulée An Illicit Whisky Still in the Highlands rappelant The Highland Whisky Still at Lochilphead de Wilkie datant de 1819. Enfin, le succès de Pitlessie Fair et Village Politicians incita Joseph William Mallord Turner (1775-1851) à peindre Country Blacksmith Disputing upon the Price of Iron and the Price Charged to the Butcher for Shoeing His Poney et Unpaid Bill, deux scènes de genre inspirées du style de Wilkie qu'il exposa en 1807 et 1808 à la Royal Academy. Selon John Walker, Turner aurait été envieux de l'attention que les collectionneurs auraient portée aux premières œuvres de Wilkie, «La Forge [...] avait [ainsi] pour but de "casser le nez" de Wilkie et pour ce faire, Turner "avait soufflé son art sur la forge du maréchal-ferrant" ${ }^{36}$ ». En 1842, avec Peace-Burial at Sea, Turner a tenu à rendre hommage au peintre écossais non pas en s'inspirant de ses scènes de genre mais imaginant les funérailles de Wilkie qui venait de décéder en mer alors qu'il rentrait d'un voyage au MoyenOrient.

32. Ibid., p. 59.

33. A. Raimbach, Memoirs and Recollections of the Late Abraham Raimbach, Esq., Engraver, Including a Memoir of Sir David Wilkie, R. A., Londres, Frederick Shoberl Junior,1843, p. 177.

34. Les toiles de Mulready intitulées The Barber's Shop (1811, œuvre non localisée), The Carpenter's Shop (1809, œuvre non localisée) et Fair-Time (1809-1839, Tate Britain, Londres) évoquent les tableaux de Wilkie. Dans Fair-Time, Mulready a même reproduit un groupe de personnages presque identiques à ceux figurant dans The Village Holiday (1809, Tate Britain, Londres) de Wilkie. Sur l'influence de Wilkie sur Mulready, voir R. et S. Redgrave, A Century of British Painters, vol. 2, Londres, Smith, Elder \& Co., 1866, p. 304.

35. W. Collins, Memoirs of the Life of William Collins, esq., R. A. With Selections from His fournals and Correspondence, vol. 1, Londres, Longman, Brown, Green et Longmans, 1848, p. 102.

36. J. Walker, Foseph Mallord William Turner, Paris, Cercle d'Art, 1980, p. 77. 
Des liens étroits ont commencé à se développer entre peinture et poésie en Écosse à partir du XVIII ${ }^{\mathrm{e}}$ siècle et n'ont pas cessé de se renforcer tout $\mathrm{au}$ long du XIX ${ }^{\mathrm{e}}$ siècle. Une étude de quelques tableaux nous a permis de constater que les peintres et les poètes s'inspiraient de thèmes identiques et qu'ils partageaient les mêmes sentiments au sujet de l'union angloécossaise. Si les poètes ont parfois été sensibles aux œuvres des peintres, l'influence des poètes sur les peintres de genre fut des plus profondes et la poésie en scots a indéniablement contribué au développement d'une peinture propre à l'Écosse. Au xx ${ }^{\mathrm{e}}$ siècle, ainsi que le rappelle le tableau Poets' Pub d'Alexander Moffat (né en 1943) représentant quelques-uns des plus éminents poètes, écrivains et critiques d'art de la seconde moitié du $\mathrm{XX}^{\mathrm{e}}$ siècle, les peintres ont continué à être influencés par les poètes ${ }^{37}$. Si désormais les peintres ne s'intéressent plus aux poèmes de Ramsay, ceux de Burns n'en finissent pas de les inspirer. En 1999, Alexander Goudie (1933-2004) a réalisé pas moins de cinquante-quatre tableaux illustrant des scènes du poème «Tam o' Shanter »; en 2013, Calum Colvin (né en 1961) s'est lui aussi inspiré des poèmes de Burns pour exécuter une série d'œuvres ${ }^{38}$. Avec ses créations artistiques, Colvin a non seulement rendu hommage à Burns mais, en reprenant dans plusieurs de ses compositions le portrait du poète peint par Alexander Nasmyth, il a aussi célébré son prédécesseur qui, comme lui, a été profondément marqué par les œuvres des poètes et hommes de lettres écossais.

\section{Références bibliographiques}

\section{Ouvrages}

Bergez Daniel, Littérature et peinture [2004], Paris, Armand Colin, 2009.

Burns Robert, Complete Poems and Songs of Robert Burns, Édimbourg, Lomond Books, 2000.

Caw Sir James, Scottish Painting Past and Present, 1620-1908 [1908], Bath, Kingsmead Reprints, 1975.

Chiego William J., Sir David Wilkie of Scotland (1785-1841), Raleigh, North Carolina Museum and Art, 1987.

Gooksey Janet C. B., Alexander Nasmyth H.R.S.A. 1758-1840. A Man of the Scottish Renaissance, Écosse, Paul Harris Whittingehame House Publishing, 1991.

37. Moffat était particulièrement proche de Hugh MacDiarmid (1892-1978).

38. Ces œuvres ont été réalisées pour l'exposition intitulée «Burnsiana qui a été présentée au Robert Burns Birthplace Museum à Alloway du 7 juin 2013 au 15 septembre 2013. 
Gunningham Allan, The Life of Sir David Wilkie with His Journals, Tours, and Critical Remarks on Works of Art and a Selection of His Correspondence, in Three Volumes, 3 vol., Londres, John Murray, 1843.

Gordon Thomas Crouther, David Allan of Alloa 1744-1796. The Scottish Hogarth, Alva, Robert Cunningham and Sons, 1951.

Grier Robertson Gordon, Scottish Scenes and Scottish Story. The Later Career of David Allan, Historical Painter, thèse de $\mathrm{PhD}$ non publiée, University of Glasgow, 1990.

Macmillan Duncan, Scottish Art 1460-2000 [1990], Édimbourg et Londres, Mainstream Publishing, 2000.

Melville Jennifer, Phillip of Spain: The Life and Art of John Phillip, 18171867, Aberdeen, Aberdeen City Council, 2005.

Ramsay Allan, The Works of Allan Ramsay, A. M. Kinghorn et A. Law (éds), 6 vols, Édimbourg et Londres, Blackwood and Sons, 1945-1974.

Thomson James, James Thomson Poetical Works, J. L. Robertson (éd.), Londres, Oxford University Press, 1951.

Tromans Nicholas (éd.), David Wilkie. Painter of Everyday Life, Londres, Dulwich Picture Gallery, 2002.

\section{Tableaux cités}

Quelques-uns des tableaux cités dans cet article peuvent être consultés sur les sites internet indiqués.

Allan David, Highland Wedding at Blair Atholl, 1780, National Gallery of Scotland, Édimbourg. Disponible sur <www.nationalgalleries.org/ collection/artists-a-z/a/artist/david-allan/object/highland-wedding-at-blair-atholl-ngl-001-81> (consulté le 17 janvier 2015).

_- Niel Gow and Donald Gow, 1780, National Gallery of Scotland, Édimbourg. Disponible sur <www.bbc.co.uk/arts/yourpaintings/paintings/ niel-gow-1727 1807-violinist-and-composer-with-his-brother212069> (consulté le 17 janvier 2015).

-, The Penny Wedding, 1795, National Gallery of Scotland, Édimbourg. Disponible sur <www.uiowa.edu/ c008103/images/pages/allenpennywedding 1795.htm> (consulté le 17 janvier 2015).

Allan Sir William, The Celebration of the Birthday of James Hogg, v. 18231825, National Gallery of Scotland, Édimbourg. Disponible sur <www. nationalgalleries.org/collection/artists-a-z/a/artist/sir-williamallan/object/the-celebration-of-the-birthday-of-james-hogg1770-1835-pg-3136> (consulté le 17 janvier 2015).

-, Heroism and Humanity: An Incident in the Life of Robert the Bruce, 1840, Art Gallery and Museum, Kelvingrove, Glasgow. Disponible sur <www. 
bbc.co.uk/arts/yourpaintings/paintings/heroism-and-humanity83047> (consulté le 17 janvier 2015).

—, The Murder of Archbishop Sharp on Magus Moor, 1679, 1821, collection particulière.

Carse Alexander, Covenanters in a Glen, v. 1800, The University of Edinburgh Fine Art Collection, Édimbourg. Disponible sur <www.bbc. co.uk/arts/yourpaintings/paintings/covenanters-in-a-glen-94045> (consulté le 17 janvier 2015).

-, The Penny Wedding, v. 1819, National Gallery of Scotland, Édimbourg. Disponible sur <www.bbc.co.uk/arts/yourpaintings/paintings/thepenny-wedding-209879> (consulté le 17 janvier 2015).

Drummond John, Tam Pursu'd by the Witches, v. 1870, The National Trust for Scotland, Robert Burns Birthplace Museum, Ayr. Disponible sur <www.bbc.co.uk/arts/yourpaintings/paintings/tam-pursud-by-thewitches-197309> (consulté le 17 janvier 2015).

Harvey George, Catechising in a Scottish School, 1832, New Walk Museum and Art Gallery, Leceister. Disponible sur <www.bbc.co.uk/arts/ yourpaintings/paintings/catechising-in-a-scottish-school-81466> (consulté le 17 janvier 2015).

—, The Christening, v. 1831, Hunterian Art Gallery, University of Glasgow. Disponible sur <www.bbc.co.uk/arts/yourpaintings/paintings/thechristening-138578> (consulté le 17 janvier 2015).

Landseer, Sir Edwin, An Illicit Whisky Still in the Highlands, Apsley House, The Wellington Museum, Londres.

Lizars William Home, A Scotch Wedding, v. 1811, National Gallery of Scotland, Édimbourg. Disponible sur <www.bbc.co.uk/arts/your paintings/paintings/a-scotch-wedding-212576> (consulté le 17 janvier 2015).

Moffat Alexander, Poets' Pub, 1980, National Gallery of Scotland, Édimbourg. Disponible sur <www.nationalgalleries.org/collection/ artists-a-z/m/artist/alexander-moffat/object/poets-pub-pg-2597> (consulté le 17 janvier 2015).

Nasmyth Alexander, Princes Street with the Commencement of the Building of the Royal Institution, 1825, National Gallery of Scotland, Édimbourg. Disponible sur <www.nationalgalleries.org/collection/artists-a-z/n/artist/ alexander-nasmyth/object/princes-street-with-the-commencementof-the-building-of-the-royal-institution-ng-2542> (consulté le 17 janvier 2015).

Pettie John, A Musician's Reverie, 1850, Aberdeen Art Gallery and Museums, Aberdeen. Disponible sur <www.bbc.co.uk/arts/yourpaintings/paintings/a-musicians-reverie-107578> (consulté le 17 janvier 2015). 
—, A Song without Words, 1859-1888, Manchester City Galleries, Manchester. Disponible sur <www.bbc.co.uk/arts/yourpaintings/paintings/a-song-without-words-205806> (consulté le 17 janvier 2015).

Phillip John, Baptism in Scotland, 1850, Aberdeen Art Gallery and Museums, Aberdeen. Disponible sur <www.bbc.co.uk/arts/yourpaintings/paintings/baptism-in-scotland-107644> (consulté le 17 janvier 2015).

-, The Music Room, McLean Museum and Art Gallery - Inverclyde Council, Greenock. Disponible sur <www.bbc.co.uk/arts/yourpaintings/paintings/the-music-room-183433> (consulté le 17 janvier 2015).

Turner Joseph Mallord William, Country Blacksmith Disputing upon the Price of Iron and the Price Charged to the Butcher for Shoeing His Poney, 1807, Tate Britain, Londres. Disponible sur <www.tate.org.uk/art/artworks/ turner-a-country-blacksmith-disputing-upon-the-price-of-iron-andthe-price-charged-to-the-n00478> (consulté le 17 janvier 2015).

-, Peace - Burial at Sea, 1842, Tate Britain, Londres. Disponible sur <www.tate.org.uk/art/artworks/turner-peace-burial-at-sea-n00528> (consulté le 17 janvier 2015).

_, Unpaid Bill, 1808, collection particulière.

Van der Goes, Hugo, The Trinity Altarpiece, v. 1478-1479, National Gallery of Scotland, Édimbourg. Disponible sur <www.nationalgalleries. org/collection/artists-a-z/g/artist/hugo-van-der-goes/object/thetrinity-altarpiece-ng-1772> (consulté le 17 janvier 2015).

WArrender Thomas, Still Life, v. 1708, National Gallery of Scotland, Édimbourg. Disponible sur <www.nationalgalleries.org/collection/ artists-a-z/w/artist/thomas-warrender/object/still-life-ng-2404> (consulté le 17 janvier 2015).

Wilkie Sir David, The Bag-Piper, 1813, Tate Britain, Londres. Disponible sur <www.tate.org.uk/art/artworks/wilkie-the-bag-piper-n00329> (consulté le 17 janvier 2015).

—, The Blind Fiddler, 1806, Tate Britain, Londres. Disponible sur <www. tate.org.uk/art/artworks/wilkie-the-blind-fiddler-n00099> (consulté le 17 janvier 2015).

- The Chelsea Pensioners Reading the Waterloo Despatch, 1822, Apsley House, The Wellington Museum, Londres. Disponible sur <www.wga.hu/ html_m/w/wilkie/chelsea.html> (consulté le 17 janvier 2015).

-, The Cottar's Saturday Night, 1837, Glasgow Art Gallery and Museum, Glasgow. Disponible sur <www.wikiart.org/en/david-wilkie/thecotter-s-saturday-night> (consulté le 17 janvier 2015).

-, The Cottage Toilet, 1824, The Wallace Collection, Londres. Disponible sur <http://wallacelive.wallacecollection.org/eMuseumPlus?service 
$=$ ExternalInterface $\&$ module $=$ collection\&objectId $=65286>$ (consulté le 17 janvier 2015).

-, Duncan Gray, 1814, Victoria and Albert Museum, Londres.

-, The Gentle Shepherd, 1823, Aberdeen Art Gallery and Museum, Aberdeen.

—, The Highland Whisky Still at Lochilphead, 1819, collection du duc de Westminster.

_, Patie Disbelieves Sir William's Prophecy from 'The Gentle Shepherd', v. 1803, collection du duc de Buccleuch et Queensberry.

_- The Penny Wedding, 1818, collection de Sa Majesté la Reine Elisabeth II. Disponible sur <www.royalcollection.org.uk/collection/405536/thepenny-wedding $>$ (consulté le 17 janvier 2015).

_- Pitlessie Fair, 1804, National Gallery of Scotland, Édimbourg. Disponible sur <www.nationalgalleries.org/collection/artists-a-z/w/artist/ sir-david-wilkie/object/pitlessie-fair-ng-1527> (consulté le 17 janvier 2015).

_- The Reading of the Will, 1820, Neue Pinakothek, Munich. Disponible en ligne sur <www.wga.hu/html_m/w/wilkie/reading.html> (consulté le 17 janvier 2015).

_, Village Politicians, 1806, collection du comte de Mansfield. 